\title{
Design of semiconducting electrides via electron-metal hybridization: the case of $\mathrm{Sc}_{2} \mathrm{C}$
}

\author{
Lauren M. McRae ${ }^{\dagger}$, Rebecca C. Radomsky ${ }^{\dagger}$, Jacob T. Pawlik ${ }^{\dagger}$, Daniel L. \\ Druffel $^{\dagger}$, Jack D. Sundberg ${ }^{\dagger}$, Matthew G. Lanetti ${ }^{\dagger}$, Carrie L. Donley*, \\ Kelly L. White ${ }^{\dagger}$, and Scott C. Warren*,†
}

$\dagger$ Department of Chemistry, University of North Carolina at Chapel Hill, Chapel Hill, North Carolina 27599, United States

\$Chapel Hill Analytical and Nanofabrication Laboratory (CHANL), University of North Carolina at Chapel Hill, Chapel Hill, North Carolina 27599, United States

Email:sw@unc.edu

\begin{abstract}
:
Electrides are exotic materials that have electrons present in well-defined lattice sites. The existence of $\mathrm{Y}_{2} \mathrm{C}$ and $\mathrm{Gd}_{2} \mathrm{C}$ as $2 \mathrm{D}$ electrides inspired us to examine other trivalent metal carbides, including $\mathrm{Sc}_{2} \mathrm{C}$ and $\mathrm{Al}_{2} \mathrm{C}$. It has been proposed that design rules for electride materials include the need for an electropositive cation adjacent to the electride site, but the effect of cation electronegativity on electronic structure in electride materials is not yet known. Here, we examine trivalent metal carbides with varying degrees of electronegativity and experimentally synthesize a $2 \mathrm{D}$ electride, $\mathrm{Sc}_{2} \mathrm{C}$, containing the most electronegative metal yet found neighboring the electride site. Further, we find that higher electronegativity of the cation drives greater hybridization between metal and electride orbitals. Our calculations predict that $\mathrm{Sc}_{2} \mathrm{C}$ is a small band gap semiconductor with a band gap of $0.305 \mathrm{eV}$, with an experimental conductivity of $1.62 \mathrm{~S} / \mathrm{cm}$ at room temperature. This is the first $2 \mathrm{D}$ electride material to exhibit semiconducting behavior, and we propose that electronegativity of the cation drives the change in band structure.
\end{abstract}

\section{Introduction:}

Challenges in energy storage, electronics, and catalysis motivate the search for exotic materials with extreme properties, and electrides - crystals with bare electrons trapped at stoichiometric concentrations $^{1-3}$ - offer some of the most exceptional. These electrons have been ejected from atomic orbitals to reside in vacant lattice sites and, because they are so weakly bound, are better electron donors than alkali metals ${ }^{4-6}$, can offer electrical conductivity that rivals silver, and can catalyze challenging reactions. These properties have led to the exploration of electrides in applications where electron-rich materials are needed: $\mathrm{N}_{2}$ and $\mathrm{CO}_{2}$ reduction ${ }^{7,8}$, battery electrodes $^{9,10}$, and electron emitters ${ }^{11-13}$. Despite this progress, rules that might predict an electride's properties based on its structure or composition are underdeveloped. For example, unlike in conventional materials, it is unknown how to tune the band gap of semiconducting 
electrides. And, even more critically, simple rules that could predict whether a material is an electride remain imprecise ${ }^{3,14}$.

Presently, just two rules for predicting electrides are widely accepted: (1) the metal atoms that form the coordination sphere around the electride electron must be electropositive ${ }^{15}$, and (2) the compound must be electron rich ${ }^{16}$. For example, in $\left[\mathrm{Ca}_{2} \mathrm{~N}\right]^{+}\left(\mathrm{e}^{-}\right)^{(17)}$, calcium is electropositive, and the compound is electron rich because the preferred oxidation states $\left(\mathrm{Ca}^{2+}, \mathrm{N}^{3-}\right)$ and stoichiometry provide an extra electron, forming an electride. These rules are far too broad, however, because many compounds fulfill these rules but are not electrides.

To improve these rules, experiments have begun to identify the metals that can be adjacent to an electride electron. So far, only $\mathrm{Ca}^{(5)}, \mathrm{Sr}^{(18,19)}, \mathrm{Ba}^{(17,20)}, \mathrm{Y}^{(21,22)}, \mathrm{Hf}^{(23)}$, and $\mathrm{Gd}^{(24,25)}$ have been incorporated next to the electride site (Figure 1), while Sc and several of the lanthanide series have been identified as candidates computationally ${ }^{26,27}$. This delicate balance between electron counting and electronegativity was recently explored in the case of $\mathrm{Ca}_{5} \mathrm{~Pb}_{3}$ (not an electride) and $\mathrm{Ca}_{3} \mathrm{Hf}_{2} \mathrm{~Pb}_{3}$ (an electride), as replacing calcium for hafnium makes the compound electron rich ${ }^{16}$. Despite these findings, the limit of cation electronegativity in the formation of electrides is not yet known. Therefore, to develop improved design rules for electrides and for their structure-property relationships, we explore the trivalent metal carbides with a layered structure. This family is informative because $\mathrm{Y}_{2} \mathrm{C}$ is a known electride ${ }^{22}, \mathrm{Sc}_{2} \mathrm{C}$ has been predicted ${ }^{26}$, and $\mathrm{Al}_{2} \mathrm{C}$ is unknown, and further, these metals vary systematically in electronegativity (Figure 1).

The question of electronegativity is essential because two experimentally observed electrides $-\mathrm{Y}_{2} \mathrm{C}$ and $\mathrm{Hf}_{2} \mathrm{~S}$ - are "hybrid" electrides ${ }^{3}$, where the electride electron has some overlap with the adjacent metal orbitals, and electronegativity of the metal may be correlated with the degree of hybridization. This covalency plays an important role in defining the electronic structure of the electride because the electride electron is normally found around the Fermi level. Thus, as

\begin{tabular}{|c|c|c|c|}
\hline \multicolumn{4}{|c|}{ Number of valence electrons } \\
\hline One & Two & Three & Four \\
\hline $\mathrm{Na}$ & $\mathrm{Mg}$ & $\mathrm{Al}$ & $\mathrm{Si}$ \\
0.93 & 1.31 & 1.61 & 1.90 \\
\hline $\mathrm{K}$ & $\mathrm{Ca}$ & $\mathrm{Sc}$ & $\mathrm{Ti}$ \\
0.82 & & 1.36 & 1.54 \\
\hline $\mathrm{Rb}$ & $\mathrm{Sr}$ & $\mathrm{Y}$ & $\mathrm{Zr}$ \\
0.82 & & & 1.33 \\
\hline $\mathrm{Cs}$ & $\mathrm{Ba}$ & $\mathrm{RE}$ & $\mathrm{Hf}$ \\
0.79 & 0.89 & & \\
\hline
\end{tabular}

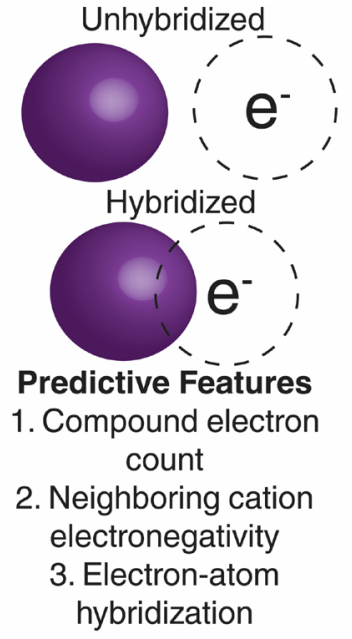

Figure 1. An adapted periodic table highlighting the valency of various cations. The electronegativity values are shown beneath each element symbol, and the gray blocks highlight the cations that have been experimentally incorporated next to an electride site, which is shown pictorially on the right. The predictive features for potential electrides that we highlight in this work are listed. An explanation of electronatom hybridization is provided pictorially. hybridization occurs, tunable band structures become possible, and indeed, $\mathrm{Y}_{2} \mathrm{C}$ is a semi-metal. This is unique among the layered electrides and raises the question of whether greater degrees of hybridization between metal and electride electron could lead to new categories of electride materials ${ }^{28}$. These questions motivate our exploration of the $\mathrm{M}_{2} \mathrm{C}$ system, and especially of layered $\mathrm{Sc}_{2} \mathrm{C}$.

To highlight uncertainties within the $\mathrm{Sc}_{2} \mathrm{C}$ system, we briefly outline previous findings and predictions. $\mathrm{Sc}_{2} \mathrm{C}$ was originally synthesized in 1967 and assigned to the $\mathrm{NaCl}$ structure type, which we refer to below as the cubic phase $^{29}$. In 1969, different authors reassigned the structure as layered 
$(R \overline{3} m)$ on the basis of its powder x-ray diffraction (PXRD) pattern ${ }^{21,30}$, even though the pattern was missing several important reflections (Table S1). In 1989, the structure was again reassigned as the cubic phase ${ }^{31}$ (Figure 2a, updated with our findings). This assignment appears to be correct and it is not known whether a layered $\mathrm{Sc}_{2} \mathrm{C}$ electride exists. Currently, a 2D $\mathrm{Sc}_{2} \mathrm{C}$ MXene structure has been explored computationally ${ }^{32,33}$ and calculations predict that layered $\mathrm{Sc}_{2} \mathrm{C}$ would be an electride ${ }^{26}$. In addition, $2 \mathrm{D} \mathrm{Sc_{2 } \mathrm { C }}$ has been predicted to store an impressive $8 \%$ hydrogen by weight $^{34,35}$ and reversibly store fluoride as a battery anode at capacities that exceed state-of-the-art lithium-ion batteries ${ }^{10}$. Thus, if a layered $\mathrm{Sc}_{2} \mathrm{C}$ electride does exist, it could allow these exciting predictions to be realized.

In this study, we predict that layered $\mathrm{Sc}_{2} \mathrm{C}$ is stable using an evolutionary algorithm and experimentally explore the phase diagram around the desired composition. We then explore the electronic structure of the synthesized material, and compare it to $\mathrm{Y}_{2} \mathrm{C}$ and $\mathrm{Al}_{2} \mathrm{C}$ to understand periodic trends. We find that as the cation becomes more electronegative, the band gap opens with $\mathrm{Y}_{2} \mathrm{C}$ as a semimetal, $\mathrm{Sc}_{2} \mathrm{C}$ as a small band gap semiconductor, and $\mathrm{Al}_{2} \mathrm{C}$ as a larger gap semiconductor. The synthesized layered $\mathrm{Sc}_{2} \mathrm{C}$ is the first $2 \mathrm{D}$ electride with semiconducting properties, opening up a fascinating new class of electride materials with several possible applications in hydrogen storage and batteries.

\section{Results and Discussion:}

Phase diagram exploration:

Although layered $\mathrm{Sc}_{2} \mathrm{C}$ is unknown, we and Hirayama et. al. ${ }^{26}$ have hypothesized that it exists because $\mathrm{Sc}$ is chemically similar to $\mathrm{Y}$ and layered $\mathrm{Y}_{2} \mathrm{C}$ is a stable phase. To investigate whether layered $\mathrm{Sc}_{2} \mathrm{C}$ is thermodynamically stable, we used USPEX to explore the scandiumcarbon phase space. The phase diagram we obtained indicates that the $\mathrm{Sc}_{2} \mathrm{C}$ composition is on the hull, and that the layered $R \overline{3} m$ structure is stable by 28 meV/atom (Figure 2b). This modest relative stability could suggest why the layered phase had not previously been synthesized.

The possibility that layered $\mathrm{Sc}_{2} \mathrm{C}$ is thermodynamically stable motivated us to make this compound via hightemperature solid-state synthesis. In the $\mathrm{Y}_{2} \mathrm{C}$ synthesis, we observed that high oxygen content in the yttrium led to formation of the cubic phase $\left(\mathrm{YC}_{\mathrm{x}}\right)$. With

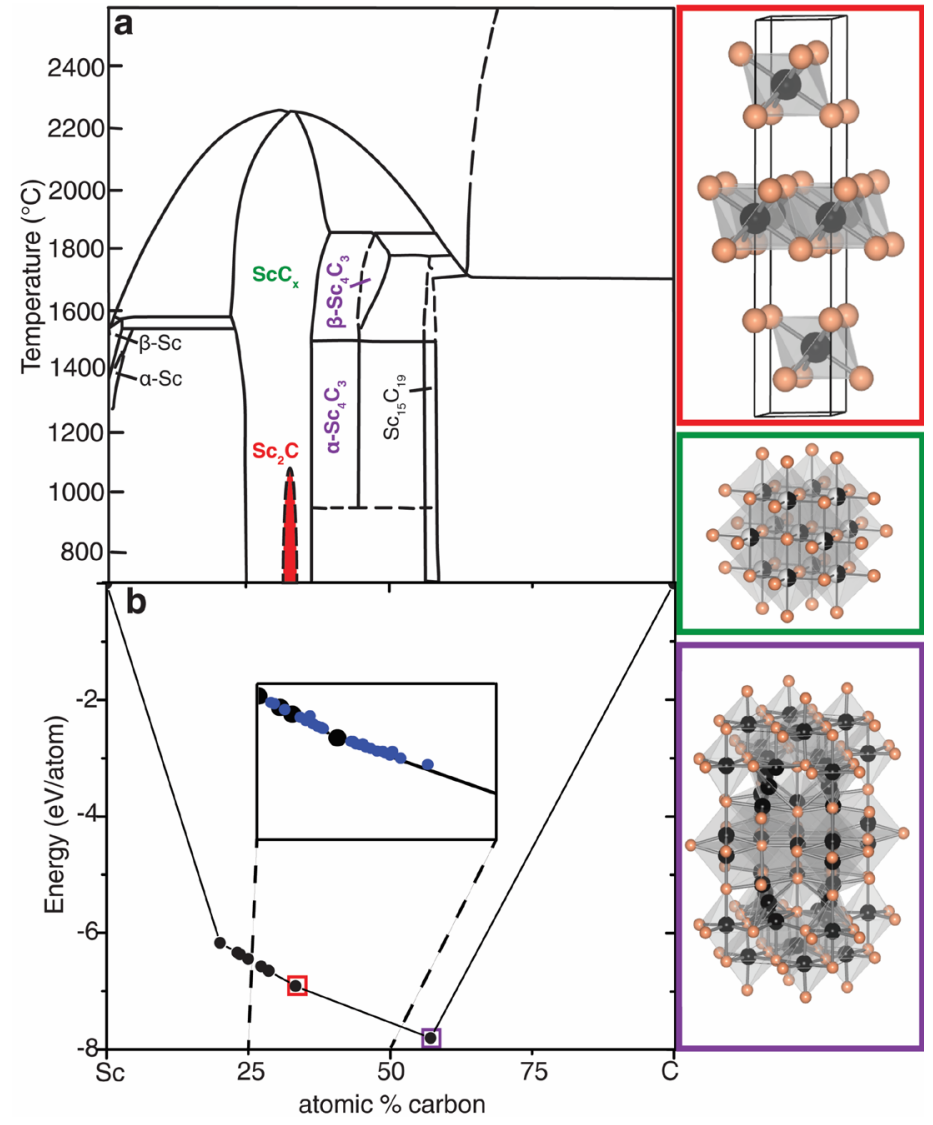

Figure 2a. The binary phase diagram of the $\mathrm{Sc}-\mathrm{C}$ system adapted from Velikanova et. al. The layered $\mathrm{Sc}_{2} \mathrm{C}$ has been incorporated (red). b. The hull diagram for the Sc-C phase calculated using USPEX. The conventional unit cell for three structures are shown corresponding to the colors on each plot. 
this in mind, compared to the synthesis performed in Rassaerts et. al.'s 1967 study, we suspected that our use of high purity scandium (with a lower oxygen content) would allow us to experimentally realize our computational prediction. We arc-melted scandium and carbon in ratios from 2:0.9 to 2:1.15, respectively. These arc-melted samples had a high content of the cubic structure $\left(\mathrm{ScC}_{\mathrm{x}}\right)$ (Figure $\left.\mathrm{S} 1\right)$, but arc-melting has a rapid cool-down, so the structure may not be at equilibrium. Therefore, we followed arc-melting with annealing for 7 days at $1100^{\circ} \mathrm{C}$ to increase the likelihood of observing the thermodynamically favored layered phase. We found that the cubic structure is stable both at compositions with deficient carbon (Figure S2) and excess carbon (Figure S3). However, at a composition of 2:0.99, the layered structure was observed, and this is reflected in the new binary phase diagram we propose for the Sc-C system (Figure $2 \mathrm{a})^{31}$. There is uncertainty in the assignment of the temperature at which the congruent layered-to-cubic phase change occurs, but our experiments indicate that the layered phase is stable to at least $1100^{\circ} \mathrm{C}$.

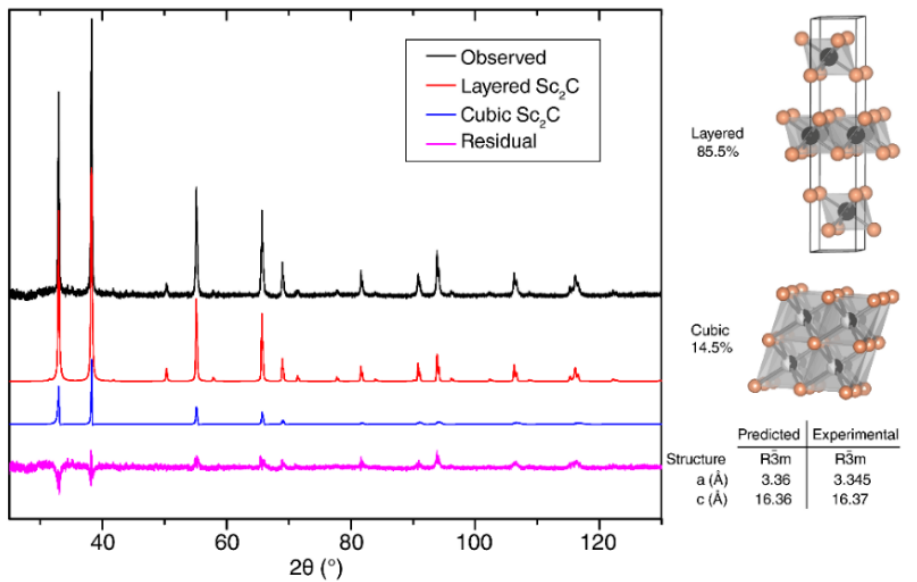

Figure 3. X-ray diffraction patterns of the experimental structure compared to the calculated layered and cubic $\mathrm{Sc}_{2} \mathrm{C}$ phases. The layered and cubic crystal structures are shown, along with the amount present calculated from the Rietveld method. The predicted and experimental lattice parameters are compared.

To fully understand the structure of the layered phase, we first performed PXRD experiments of the product at room temperature and refined the pattern using the Rietveld method $^{36}$ (Figure 3). The pattern indexed as the $R \overline{3} m$ structure with lattice parameters of $a=b=3.345$ (2) $\AA$ and $c=16.37$ (2) $\AA$. This refinement includes the incorporation of preferred orientation at $\{104\}$ with a March coefficient of 0.754 . The sample contained up to $15 \%$ of the cubic $\mathrm{ScC}_{\mathrm{x}}$, which we attribute to a relatively low stability of the layered phase. To quantitatively compare the predicted and experimental structures, we further optimized the structure from USPEX using the PBEsol functional. The calculated lattice parameters were $0.45 \%$ smaller in $a$ and $0.06 \%$ smaller in $c$, which is less than the typical error between PBEsol and experiment. This further confirms that the synthesized material is layered $\mathrm{Sc}_{2} \mathrm{C}$.

To better understand the effect of annealing in these samples, we performed transmission electron microscopy (TEM) of the product at room temperature under high vacuum. In Figure 4, we compare the Fast Fourier Transform (FFT) of these samples, where the non-annealed sample shows a ring pattern while the annealed sample shows well-defined spots. From the FFT images, we constructed filtered high resolution-TEM (HRTEM) images to create a color-coded map of crystalline domains where we compare the crystallinity of $\mathrm{Sc}_{2} \mathrm{C}$ before and after annealing. The filtered HRTEM images and FFTs confirm an increase in domain size upon annealing, likely due to kinetic trapping from rapid cooling after arc-melting.

In addition, we used TEM to confirm that the layered $\mathrm{Sc}_{2} \mathrm{C}$ phase is dominant. While from $\mathrm{XRD}$ there is a small amount of cubic $\mathrm{Sc}_{2} \mathrm{C}(14.5 \%)$ compared to layered $\mathrm{Sc}_{2} \mathrm{C}(85.5 \%)$, the 
refinement has some error because the layered and cubic patterns are similar. In electron diffraction, the most common spatial frequency corresponds to a d-spacing of $2.3 \AA$, which we assign to either $\{104\}$ in the layered structure or $\{101\}$ in the cubic structure. This ambiguity motivated us to find distinct peaks for the layered phase. To identify the phase of these domains, we sought to acquire diffraction patterns containing the $\{117\}$ reflection of the layered phase, which is expected at $5.501 \mathrm{~nm}^{-1}$, for which there are no reflections in the cubic phase. In Figure $4 \mathrm{c}$, we observe diffraction spots at $5.479 \mathrm{~nm}^{-1}$, which can only be assigned to the layered phase. Simulations of the cubic structure with varied arrangements of carbon also failed to show a diffraction peak at $5.501 \mathrm{~nm}^{-1}$. Importantly, the prevalence of $\{117\}$ in diffraction patterns increases upon annealing, in agreement with XRD. In addition, XRD and TEM lattice spacings agree within $0.6 \%$. This data confirms the experimental structure from XRD and corroborates the Rietveld refinement result.
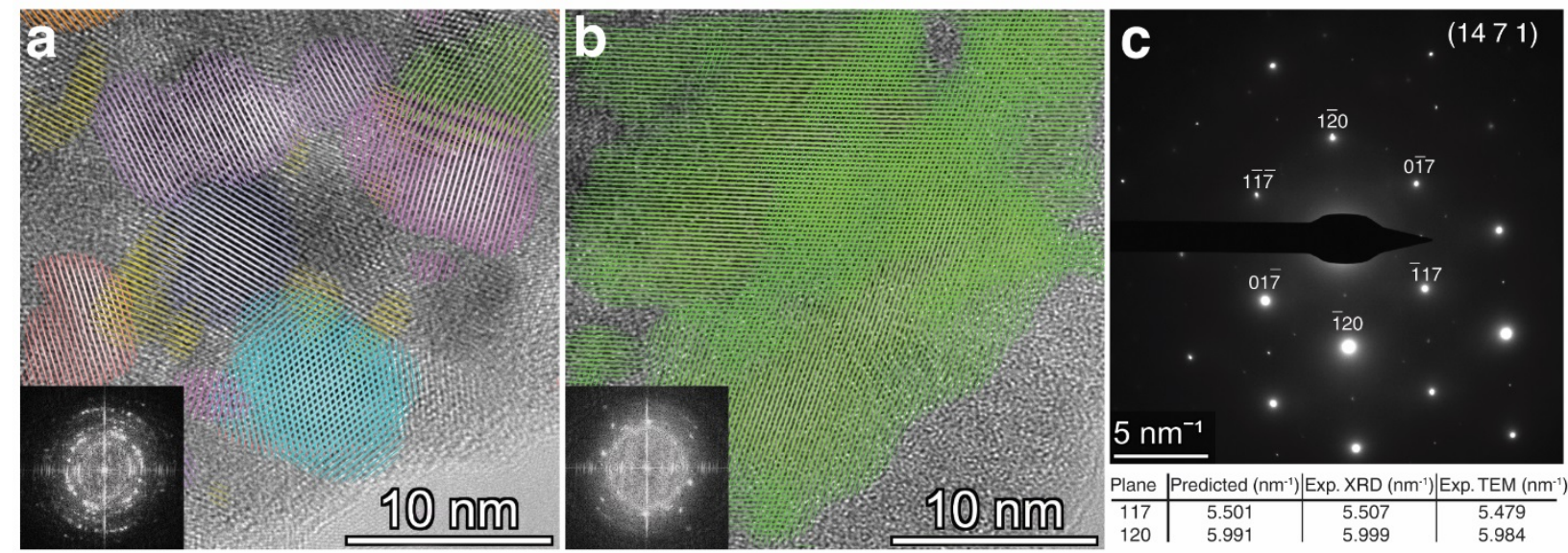

Figure 4a. A high-resolution TEM image of Sc2C before annealing, with colored lattice planes corresponding to different crystallite regions determined through FFTs. b. A high-resolution TEM image of Sc2C after annealing for 7 days, with the lattice planes of the single crystallite region in green. c. A selected area electron diffraction (SAED) pattern of the $(14,7,1)$ zone axis. The corresponding predicted and experimental d-spacings are compared.

\section{Assessment of electride character and electronic structure:}

Having confirmed the existence of layered $\mathrm{Sc}_{2} \mathrm{C}$ as a thermodynamically stable material, we examined the material's electride character. In the experimental structure determined from our Rietveld refinement, there was a longer scandium-scandium distance $(3.53 \AA)$ compared to scandium metal $(3.22 \AA)$ and $\mathrm{Sc}_{2} \mathrm{O}_{3}(3.27 \AA)$. This increased distance could indicate that electride electrons are present, so we sought evidence by calculating the electron localization function (ELF) using the hybrid DFT functional HSE-06. The ELF measures the degree of electron spatial localization compared to a reference electron with the same spin, and provides a map of electron probability across a system. The ELF of $\mathrm{Sc}_{2} \mathrm{C}$ shows localization of electrons that are not 


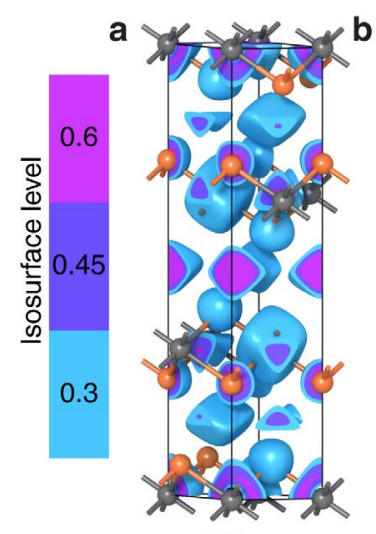

(s) C
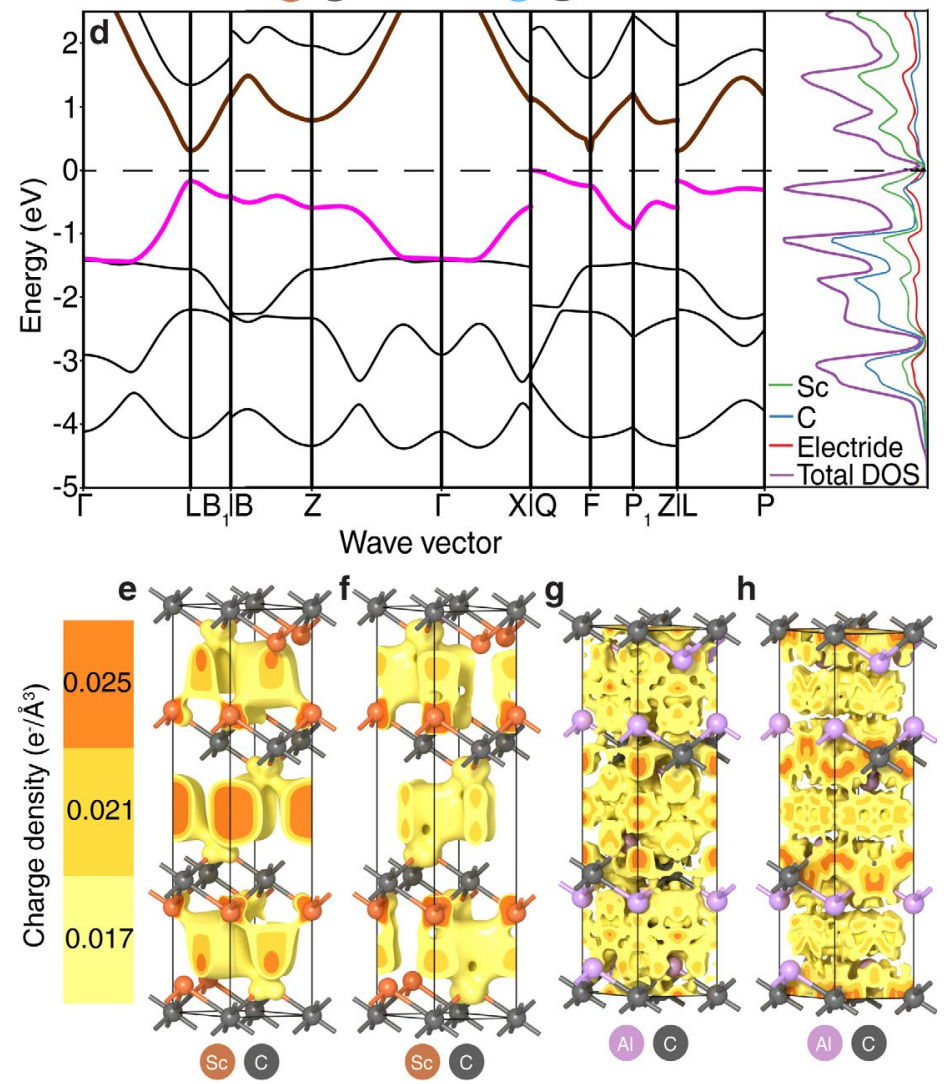

Figure 5a. The 3D ELF of $\mathrm{Sc}_{2} \mathrm{C}$, where the gradient of isosurface level spans from 0.3-0.6. This scale is the same for each ELF. b. The 3D ELF of $\mathrm{Y}_{2} \mathrm{C}$. c. The 3D ELF of $\mathrm{Al}_{2} \mathrm{C}$. d. The band structure and projected density of states of $\mathrm{Sc}_{2} \mathrm{C}$, where the valence and conduction bands are highlighted in pink and brown, respectively. e. The charge density of the $\mathrm{Sc}_{2} \mathrm{C}$ valence band at the $\mathrm{L}$ point. The gradient of charge density spans from $0.017 \mathrm{e}^{-} / \AA^{3}-0.025 \mathrm{e}^{-} / \AA^{3}$. This scale is the same for each charge density. f. The charge density of the $\mathrm{Sc}_{2} \mathrm{C}$ conduction band at the $\mathrm{L}$ point. $\mathrm{g}$. The charge density of the $\mathrm{Al}_{2} \mathrm{C}$ valence band at the $\mathrm{L}$ point. $\mathbf{h}$. The charge density of the $\mathrm{Al}_{2} \mathrm{C}$ conduction band at the $\mathrm{L}$ point. associated with either the scandium or carbon atoms, and indicates that there is electride character in the layered structure (Figure 5a).

We calculated the band structure and projected density of states (pDOS) with HSE-06 and found that $\mathrm{Sc}_{2} \mathrm{C}$ is a semiconductor with a $0.305 \mathrm{eV}$ indirect band gap and a $0.476 \mathrm{eV}$ direct band gap (Figure 5d). The pDOS was calculated with a dummy atom of no charge to illustrate the contribution of the electride states to the overall density. This shows that the electride electron density mirrors that of the scandium and that the scandium orbitals are hybridizing with the electride electron density in the same way that has been observed in $\mathrm{Y}_{2} \mathrm{C}$ and $\mathrm{Hf}_{2} \mathrm{~S}^{3}$.

We measured the electrical conductivity to confirm these predictions and further corroborate the match between experiment and calculations. Using the AMSET package $^{37}$, we predicted the conductivity would be $22.2 \mathrm{~S} / \mathrm{cm}$ at $28.4^{\circ} \mathrm{C}$ using the band structure of $\mathrm{Sc}_{2} \mathrm{C}$. We performed conductivity measurements at the same temperature, where we used a well-insulated system to accurately measure temperature. We found that the conductivity was 1.62 $\mathrm{S} / \mathrm{cm}$, which is an order of magnitude smaller than the value we calculated; however, we expect the calculation to overestimate the conductivity because it does not include impurities in the crystal, which are present in experiment. This conductivity confirms that the synthesized material is indeed a semiconductor with a small band gap. 


\section{Emergence of semiconducting character in electrides}

To understand how cation electronegativity plays a role in band structure, we compare these results to the electronic structure of $\mathrm{Y}_{2} \mathrm{C}$ and $\mathrm{Al}_{2} \mathrm{C}$. The known hybrid electride $\mathrm{Y}_{2} \mathrm{C}$ has a similar ELF to $\mathrm{Sc}_{2} \mathrm{C}$, where there is electron character that is not associated with either the yttrium or carbon atoms (Figure 5b). Yttrium has an electronegativity value of 1.22, whereas scandium has a value of $1.36^{(38)}$. Therefore, $\mathrm{Sc}_{2} \mathrm{C}$ is the $2 \mathrm{D}$ electride with the most electronegative metal adjacent to the electride site yet synthesized. We wanted to further explore a possible electride structure with a similar, yet more electronegative metal, aluminum (1.61). A layered $\mathrm{Al}_{2} \mathrm{C}$ has a similar structure to that of both $\mathrm{Y}_{2} \mathrm{C}$ and $\mathrm{Sc}_{2} \mathrm{C}$ with a space group of $R \overline{3} m$, and there is observed electron localization in this structure between the layers of $\mathrm{Al}_{2} \mathrm{C}$ (Figure 5c). We calculated band structures and band gap of $\mathrm{Y}_{2} \mathrm{C}$ (Figure $\mathrm{S} 5$ ), $\mathrm{Sc}_{2} \mathrm{C}$, and $\mathrm{Al}_{2} \mathrm{C}$ (Figure $\mathrm{S} 6$ ) using the same calculation parameters. $\mathrm{Y}_{2} \mathrm{C}$ is a semimetal, while $\mathrm{Sc}_{2} \mathrm{C}$ and $\mathrm{Al}_{2} \mathrm{C}$ are indirect band gap semiconductors, where the band gap increases from 0.31 to $0.50 \mathrm{eV}$ as the electronegativity of the cation increases.

The metal-metal distances in these $\mathrm{M}_{2} \mathrm{C}$ systems are all increased with respect to their metallic counterparts, and compared to one another, the $\mathrm{Y}-\mathrm{Y}$ distance is the largest (3.96 $\AA$ ), ScSc is in the middle (3.53 $\AA$ ), and Al-Al is the shortest (3.09 $\AA$ ). Although one expects a different ionic radius for each cation, the metal-metal distances span such a large range that the differences in metal ionic radii are negligible; we conclude there is an increase in metal-electron hybridization from $\mathrm{Y}$ to $\mathrm{Sc}$ to $\mathrm{Al}$. From these observations, we propose a model in which increasing electronegativity of metal leads to increased hybridization between unoccupied metal orbitals and the electride electron. This hybridization is because of similar energies and because of spatial proximity. Increased hybridization between electride state and empty orbitals leads to greater splitting between the filled valence band and empty conduction band - and thus a larger band gap. The relatively large amount of hybridization in the $\mathrm{Al}_{2} \mathrm{C}$ structure would lead to a small amount of electron localization. $\mathrm{Al}_{2} \mathrm{C}$ as a low electride character species supports the idea that neighboring cation electronegativity plays a role in materials exhibiting electride character, and the limit of cation electronegativity in electride materials likely lies somewhere just below the electronegativity value of aluminum.

If this model is correct, we should observe electride character in both the valence and conduction bands. Thus, we examined charge density at the top of the valence band and bottom of the conduction band in $\mathrm{Sc}_{2} \mathrm{C}$ and $\mathrm{Al}_{2} \mathrm{C}$ (Figures 5e-h). This indeed revealed electride character in both states, which could only result from hybridization of the electride electron and empty metal orbitals. This hybridization is analogous to a donor-acceptor interaction in molecules, such as $\mathrm{BH}_{3}-$ $\mathrm{NH}_{3}$, but with the $2 \mathrm{D}$ electron layer playing the role of the donor and the $2 \mathrm{D} \mathrm{M}_{2} \mathrm{C}$ atoms playing the role of acceptor. Therefore, as overlap increases between donor (electride electrons) and acceptor (empty metal-carbide orbitals), the valence and conduction bands split more, and the band gap increases. Presumably, the greatest hybridization seen in the $\mathrm{Al}_{2} \mathrm{C}$ system is facilitated by aluminum being most electronegative, since the empty $\mathrm{Al}$ orbitals are closer in energy to the electride electrons. This directly leads us to a powerful design rule for layered semiconducting electrides: that increasing metal electronegativity leads to increased electron-metal hybridization, yielding an increased band gap. 


\section{Exfoliation potential:}

To assess anisotropy in the layered $\mathrm{Sc}_{2} \mathrm{C}$, we computationally predicted the work function using the thin slab method ${ }^{39}$. We found that $\mathrm{Sc}_{2} \mathrm{C}$ has a work function of $3.78 \mathrm{eV}$ for the (0001) plane (Figure S7) and $2.89 \mathrm{eV}$ for the (1120) plane (Figure S8), which are comparable to the $\mathrm{Y}_{2} \mathrm{C}$ values of $3.72 \mathrm{eV}$ and $2.84 \mathrm{eV}$ at $(0001)$ and $(11 \overline{2} 0)$, respectively ${ }^{22}$. The change in work function in different directions shows the anisotropic nature of $\mathrm{Sc}_{2} \mathrm{C}$. The similar work functions of $\mathrm{Sc}_{2} \mathrm{C}$ and $\mathrm{Y}_{2} \mathrm{C}$ support their similarity, but $\mathrm{Sc}_{2} \mathrm{C}$ has a slightly larger work function, consistent with greater electronegativity of scandium. Nevertheless, $\mathrm{Sc}_{2} \mathrm{C}$ is still a good reducing agent. To explore anisotropy in a different way, we calculated the interlayer and intralayer stiffness tensors of $\mathrm{Sc}_{2} \mathrm{C}$ to be $136.7 \mathrm{GPa}$ and $286.8 \mathrm{GPa}$. Compared to $\mathrm{Y}_{2} \mathrm{C}$, these values indicate a greater degree of anisotropy because the intralayer stiffness tensor of $\mathrm{Sc}_{2} \mathrm{C}$ is $110 \%$ greater than its interlayer counterpart, whereas the intralayer tensor of $\mathrm{Y}_{2} \mathrm{C}$ is only $44 \%$ larger ${ }^{40}$ (Table S5).

Additionally, we calculated the energy to separate two monolayers of $\mathrm{Sc}_{2} \mathrm{C}$ (i.e. binding energy). We obtained a value of $2.76 \mathrm{~J} / \mathrm{m}^{2}$, which is larger than that of $\mathrm{Y}_{2} \mathrm{C}^{41}$. Compared to other known layered electrides with similar structures $\left(\mathrm{Ca}_{2} \mathrm{~N}\right.$ and $\left.\mathrm{Sr}_{2} \mathrm{~N}\right)$, they exhibit a similar trend where the electride with the smaller cation, $\mathrm{Ca}_{2} \mathrm{~N}$, has a larger binding energy than $\mathrm{Sr}_{2} \mathrm{~N}$. This is likely due to the smaller cation electride having a smaller interlayer distance, giving a higher electrostatic attraction between the layers. The larger binding energy of the carbide systems overall is likely due to additional covalency, which is present in the nitride systems to a lesser degree.

Finally, we calculated the mechanical properties of $\mathrm{Sc}_{2} \mathrm{C}$ as compared to $\mathrm{Y}_{2} \mathrm{C}$ (Table $\mathrm{S} 6$ ). We found that $\mathrm{Sc}_{2} \mathrm{C}$ has a higher bulk modulus than $\mathrm{Y}_{2} \mathrm{C}$, while it's shear modulus and Young's modulus are both lower. This indicates that $\mathrm{Sc}_{2} \mathrm{C}$ is more resistant to compression than $\mathrm{Y}_{2} \mathrm{C}, 395.6$ but its strain response to stress is much higher. The higher bulk modulus, lower shear modulus, and higher degree of anisotropy indicate that the material has a higher exfoliation potential than $\mathrm{Y}_{2} \mathrm{C}$. We explored this experimentally, and found that the material wouldn't exfoliate after sonicating in 1,3-dioxolane for 800 minutes. SAED patterns and TEM images show that no nanosheets or aggregates are present (Figure S9). Nevertheless, experimental exfoliation could lead to realization of many exciting applications proposed for $2 \mathrm{D} \mathrm{Sc}_{2} \mathrm{C}$, and these calculations predict that it should be explored further. 


\section{Stability in air:}

Finally, we tested the stability of this novel material in air and further explored the effect of oxidation on the crystal structure of $\mathrm{Sc}_{2} \mathrm{C}$. We performed PXRD experiments, taking data at various timepoints after letting the product sit in air (Figure 6). The data shows that the material oxidizes slowly, with almost no change after sitting in air for one day. However, after four days, there was a shift to higher angles, decreased intensity, and increased width. These attributes indicate a decrease in crystallinity as more oxygen

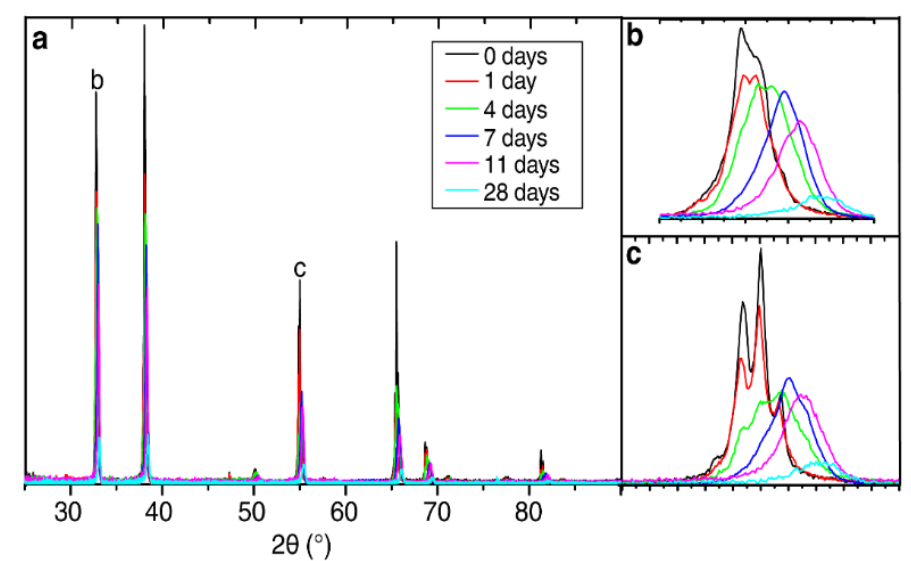

Figure 6a. X-ray diffraction patterns after various amounts of oxidation. $\mathbf{b}$. The diffraction pattern zoomed in between $54-56^{\circ}$ two-theta. c. The diffraction pattern zoomed in between $32.4-33.4^{\circ}$ two-theta. incorporates. This correlates with formation of an amorphous oxide, which is the dominant phase by the final timepoint, where there is little intensity throughout the diffraction pattern. The particle size is, on average, greater than 1 $\mu \mathrm{m}$ (Figure S9), indicating that the oxidation process isn't self-limiting.

To better understand the mechanism of oxidation, we performed x-ray photoelectron spectroscopy (XPS). High-resolution scans of scandium and carbon were taken at 0 days, 1 day, and 11 days sitting in air (Figure 7). Scandium shows four species at the initial timepoint, indicating several scandium species were present. Component A corresponds to $\left(\mathrm{Sc}^{3+}\right)^{42}$, likely due to surface oxidation during transfer into the XPS. We observe one species (component B) at binding energy that were previously assigned to $\left(\mathrm{Sc}^{2+}\right)^{43}$. We assume that component $\mathrm{C}$ is an intermediate species as the carbide transitions to the fully formed oxide, with peaks that lie between the component A and B doublets. Finally, there is a small peak with a low binding energy (395.6 $\mathrm{eV}$ ), where it is unclear whether this is scandium or possibly nitrogen as the binding energy of peak is low for either element. There are four species at the initial timepoint for carbon, where component $\mathrm{E}$ and $\mathrm{G}$ are adventitious carbon and its oxidized counterpart, respectively ${ }^{44}$. Component $\mathrm{F}$ is likely the carbide species, as peaks at a similar shift have been seen in other transition metal carbide species ${ }^{45}$. The carbide peak energy in this material is relatively reduced compared to other known transition metal carbide species, which is not surprising from an electride. Component $\mathrm{H}$ is likely an intermediate species as the carbide oxidizes. After one day of exposure to air, the $\mathrm{Sc}^{2+}$ peak decreases, the $\mathrm{Sc}^{3+}$ peak increases, and the carbide carbon peak decreases in intensity, indicating the sample is oxidizing. In the carbon spectra, a new species, 
Scandium

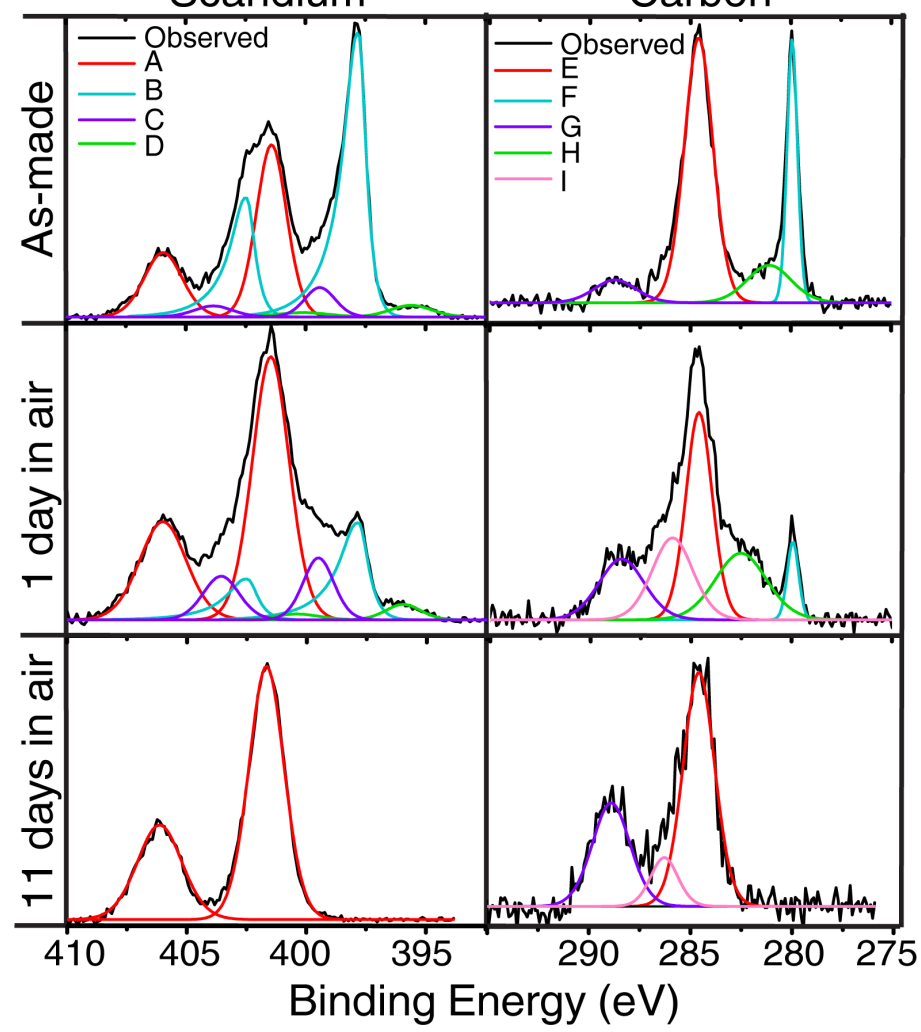

component I, becomes apparent, and likely results from the adventitious carbon oxidizing. At the 11-day timepoint, the peaks associated with $\mathrm{Sc}_{2} \mathrm{C}$ in both the scandium and carbon spectra have disappeared, indicating the surface was completely oxidized. The XRD data shows that there are still crystalline peaks present at the 11-day timepoint. From XRD and XPS, we conclude that an amorphous oxide forms on the surface of crystals first, and as the material continues to be exposed to air, oxygen diffuses further into the crystals until the bulk material becomes an amorphous oxide after 28 days. Therefore, handling this material in air will require passivating coatings.

Figure 7. X-ray photoelectron spectroscopy spectra of scandium and carbon as-made, after 1 day in air, and after 11 days in air.

\section{Conclusions}

We have synthesized a new $2 \mathrm{D}$ inorganic electride, $\mathrm{Sc}_{2} \mathrm{C}$, updating the previously published $\mathrm{Sc}-\mathrm{C}$ phase diagram. This electride includes the most electronegative cation adjacent to the electride site. We determine that while there needs to be an electropositive cation present in the structure next to the electride site, the upper limit of electronegativity likely falls just above that of aluminum. We find that this material is the first synthesized small band gap semiconductor 2D electride. Along with our exploration of $\mathrm{Al}_{2} \mathrm{C}$, the trivalent metal carbides lead to a new design rule for semiconducting $2 \mathrm{D}$ electrides: that more electronegative cations in $2 \mathrm{D}$ electrides lead to higher hybridization of the electride electron with the metal, resulting in greater splitting of valence and conduction bands. Therefore, based on the electronegativity of the cation, the band gap in these electride materials can be tuned. The small band gap and good conductivity also allow applications as a battery electrode or in IR photodetectors. Semiconducting electrides under photoexcitation could exhibit both interesting reduction and oxidation capabilities, which could be useful for photocatalysis or catalysis. The experimental realization of layered $\mathrm{Sc}_{2} \mathrm{C}$ will allow predictions of its enormous $\mathrm{H}_{2}$ and $\mathrm{F}^{-}$storage capacities to be explored.

\section{$\underline{\text { Acknowledgement }}$}


S.C.W. acknowledges support of this research by NSF grant DMR-1905294. L.M.M. and R.C.R. acknowledge support by the NSF Graduate Research Fellowship (GRF) under grant DGE1650116. J.T.P. and J.D.S acknowledge support by the NSF GRF under grant DGE-1650114. M.G.L. acknowledges support by the Department of Defense (DoD) through the National Defense Science \& Engineering Graduate Fellowship (NDSEG). This work was performed in part at the Chapel Hill Analytical and Nanofabrication Laboratory, CHANL, a member of the North Carolina Research Triangle Nanotechnology Network, RTNN, which is supported by the National Science Foundation, Grant ECCS-2025064, as part of the National Nanotechnology Coordinated Infrastructure, NNCI. Computational resources were provided, in part, by the Research Computing Center at the University of North Carolina at Chapel Hill. We would like to acknowledge Dylan Storan, a visiting scholar from the University of Limerick, for his insights on various conductivity measurements.

\section{Supporting Information Available}

Additional details regarding the literature search, computational and experimental methods, and experimental observations.

\section{$\underline{\text { References }}$}

(1) Matsuishi, S., Toda, Y., Miyakawa, M., Hayashi, K., Kamiya, T., Hirano, M., Tanaka, I., Hosono, H. High-Density Electron Anions in a Nanoporous Single Crystal : [ $\mathrm{Ca} \wedge \mathrm{Sub}$... Science (80-. ). 2003, 301, 626-630.

(2) Dye, J. L. Electrides: Early Examples of Quantum Confinement. Acc. Chem. Res. 2009, 42 (10), 1564-1572. https://doi.org/10.1021/ar9000857.

(3) Hosono, H.; Kitano, M. Advances in Materials and Applications of Inorganic Electrides. Chem. Rev. 2021, 121, 3121-3185. https://doi.org/10.1021/acs.chemrev.0c01071.

(4) Toda, Y.; Yanagi, H.; Ikenaga, E.; Kim, J. J.; Kobata, M.; Ueda, S.; Kamiya, T.; Hirano, M.; Kobayashi, K.; Hosono, H. Work Function of a Room-Temperature, Stable Electride [Ca 24A128064]4+(e-) 4. Adv. Mater. 2007, 19 (21), 3564-3569.

https://doi.org/10.1002/adma.200700663.

(5) Gregory, D. H.; Bowman, A.; Baker, C. F.; Weston, D. P. Dicalcium Nitride, Ca2N - A 2D "excess Electron" Compound; Synthetic Rotates and Crystal Chemistry. J. Mater. Chem. 2000, 10 (7), 1635-1641. https://doi.org/10.1039/b001911i.

(6) Zhang, X.; Yang, G. Recent Advances and Applications of Inorganic Electrides. J. Phys. Chem. Lett. 2020, 11 (10), 3841-3852. https://doi.org/10.1021/acs.jpclett.0c00671.

(7) Kuganathan, N.; Hosono, H.; Shluger, A. L.; Sushko, P. V. Enhanced N2 Dissociation on Ru-Loaded Inorganic Electride. J. Am. Chem. Soc. 2014, 136 (6), 2216-2219. https://doi.org/10.1021/ja410925g.

(8) Qi, M.; Tang, C.; Zhou, Z.; Ma, F.; Mo, Y. Electride-Sponsored Radical-Controlled CO2 Reduction to Organic Acids: A Computational Design. Chem. - A Eur. J. 2020, 26 (28), 6234-6239. https://doi.org/10.1002/chem.202000092. 
(9) Hu, J.; Xu, B.; Yang, S. A.; Guan, S.; Ouyang, C.; Yao, Y. 2D Electrides as Promising Anode Materials for Na-Ion Batteries from First-Principles Study. ACS Appl. Mater. Interfaces 2015, 7 (43), 24016-24022. https://doi.org/10.1021/acsami.5b06847.

(10) Druffel, D. L.; Pawlik, J. T.; Sundberg, J. D.; McRae, L. M.; Lanetti, M. G.; Warren, S. C. First-Principles Prediction of Electrochemical Electron-Anion Exchange: Ion Insertion without Redox. J. Phys. Chem. Lett. 2020, No. 1, 9210-9214. https://doi.org/10.1021/acs.jpclett.0c02266.

(11) Toda, Y.; Matsuishi, S.; Hayashi, K.; Ueda, K.; Kamiya, T.; Hirano, M.; Hosono, H. Field Emission of Electron Anions Clathrated in Subnanometer-Sized Cages in [Ca24A128064]4+(4e-). Adv. Mater. 2004, 16 (8), 685-689. https://doi.org/10.1002/adma.200306484.

(12) Hosono, H.; Hayashi, K.; Kamiya, T.; Atou, T.; Susaki, T. New Functionalities in Abundant Element Oxides: Ubiquitous Element Strategy. Sci. Technol. Adv. Mater. 2011, 12 (3). https://doi.org/10.1088/1468-6996/12/3/034303.

(13) Menamparambath, M. M.; Park, J. H.; Yoo, H. S.; Patole, S. P.; Yoo, J. B.; Kim, S. W.; Baik, S. Large Work Function Difference Driven Electron Transfer from Electrides to Single-Walled Carbon Nanotubes. Nanoscale 2014, 6 (15), 8844-8851. https://doi.org/10.1039/c4nr01629g.

(14) Dale, S. G.; Johnson, E. R. Theoretical Descriptors of Electrides. J. Phys. Chem. A 2018, 122 (49), 9371-9391. https://doi.org/10.1021/acs.jpca.8b08548.

(15) Redko, M. Y.; Jackson, J. E.; Huang, R. H.; Dye, J. L. Design and Synthesis of a Thermally Stable Organic Electride. J. Am. Chem. Soc. 2005, 127 (35), 12416-12422. https://doi.org/10.1021/ja053216f.

(16) Li, K.; Gong, Y.; Wang, J.; Hosono, H. Electron-Deficient-Type Electride Ca5Pb3 : Extension of Electride Chemical Space. J. Am. Chem. Soc. 2021, 143 (23), 8821-8828. https://doi.org/10.1021/jacs.1c03278.

(17) Walsh, A.; Scanlon, D. O. Electron Excess in Alkaline Earth Sub-Nitrides: 2D Electron Gas or 3D Electride? J. Mater. Chem. C 2013, 1 (22), 3525-3528. https://doi.org/10.1039/c3tc30690a.

(18) Brese, N. E.; O’Keeffe, M. Synthesis , Crystal and Physical Properties of Sr2N. J. Solid State Chem. 1990, 87, 134-140.

(19) Chanhom, P.; Fritz, K. E.; Burton, L. A.; Kloppenburg, J.; Filinchuk, Y.; Senyshyn, A.; Wang, M.; Feng, Z.; Insin, N.; Suntivich, J.; et al. Sr3CrN3: A New Electride with a Partially Filled d-Shell Transition Metal. J. Am. Chem. Soc. 2019, 141 (27), 10595-10598. https://doi.org/10.1021/jacs.9b03472.

(20) Reckeweg, O.; DiSalvo, F. J. Crystal Structure of Dibarium Mononitride, Ba2N, an Alkaline Earth Metal Subnitride. Zeitschrift fur Krist. - New Cryst. Struct. 2005, 220 (14), 549-550. https://doi.org/10.1524/ncrs.2005.220.14.549.

(21) Atoji, M.; Kikuchi, M. Crystal Structures of Cubic and Trigonal Yttrium Hypocarbides; A Dimorphically Interphased Single-Crystal Study. J. Chem. Phys. 1969, 51 (9), 3863-3872. 
https://doi.org/10.1063/1.1672603.

(22) Zhang, X.; Xiao, Z.; Lei, H.; Toda, Y.; Matsuishi, S.; Kamiya, T.; Ueda, S.; Hosono, H. Two-Dimensional Transition-Metal Electride Y 2 C. Chem. Mater. 2014, 26 (22), 66386643. https://doi.org/10.1021/cm503512h.

(23) Kang, S. H.; Kang, S. H.; Bang, J.; Chung, K.; Nandadasa, C. N.; Han, G.; Lee, S.; Lee, K. H.; Lee, K.; Ma, Y.; et al. Water- And Acid-Stable Self-Passivated Dihafnium Sulfide Electride and Its Persistent Electrocatalytic Reaction. Sci. Adv. 2020, 6 (23). https://doi.org/10.1126/sciadv.aba7416.

(24) Mudryk, Y.; Paudyal, D.; Pecharsky, V. K.; Gschneidner, K. A. Magnetic Properties of Gd2C: Experiment and First Principles Calculations. J. Appl. Phys. 2011, 109 (7), 2-5. https://doi.org/10.1063/1.3554257.

(25) Lee, S. Y.; Hwang, J. Y.; Park, J.; Nandadasa, C. N.; Kim, Y.; Bang, J.; Lee, K.; Lee, K. H.; Zhang, Y.; Ma, Y.; et al. Ferromagnetic Quasi-Atomic Electrons in Two-Dimensional Electride. Nat. Commun. 2020, 11 (1), 1-8. https://doi.org/10.1038/s41467-020-15253-5.

(26) Hirayama, M.; Matsuishi, S.; Hosono, H.; Murakami, S. Electrides as a New Platform of Topological Materials. Phys. Rev. X 2018, 8 (3), 31067. https://doi.org/10.1103/PhysRevX.8.031067.

(27) Inoshita, T.; Jeong, S.; Hamada, N.; Hosono, H. Exploration for Two-Dimensional Electrides via Database Screening and Ab Initio Alculation. Phys. Rev. X 2014, 4 (3), 1-8. https://doi.org/10.1103/PhysRevX.4.031023.

(28) Park, J.; Hwang, J. Y.; Lee, K. H.; Kim, S. G.; Lee, K.; Kim, S. W. Tuning the SpinAlignment of Interstitial Electrons in Two-Dimensional Y2C Electride via Chemical Pressure. J. Am. Chem. Soc. 2017, 139 (48), 17277-17280. https://doi.org/10.1021/jacs.7b10338.

(29) Rassaerts, H.; Nowotny, H.; Vinek, G.; Benesovsky, F. Zum System Scandium. Inorganic, Struct. Phys. Chem. 1967, 61, 460-468.

(30) Krikorian, N. H.; Bowman, A. L.; Krupka, M. C.; Arnold, G. P. The Preparation and Crystal Structure of Sc4C3. High Temp. Sci. 1969, 1, 360-366.

(31) Velikanova, T. Y.; Eremenko, V. N.; Artyukh, L. V.; Bondar, A. A.; Gordiichuk, O. V. Methods of Investigation and Properties of Powder Metallurgy Materials: Phase Diagrams of Sc-M(IV-VII)-C Systems. Sov. Powder Metall. Met. Ceram. 1989, 28, 711-718.

(32) Khazaei, M.; Arai, M.; Sasaki, T.; Chung, C. Y.; Venkataramanan, N. S.; Estili, M.; Sakka, Y.; Kawazoe, Y. Novel Electronic and Magnetic Properties of Two-Dimensional Transition Metal Carbides and Nitrides. Adv. Funct. Mater. 2013, 23 (17), 2185-2192. https://doi.org/10.1002/adfm.201202502.

(33) Bae, S.; Espinosa-García, W.; Kang, Y. G.; Egawa, N.; Lee, J.; Kuwahata, K.; Khazaei, M.; Ohno, K.; Kim, Y. H.; Han, M. J.; et al. MXene Phase with C3 Structure Unit: A Family of 2D Electrides. Adv. Funct. Mater. 2021, 2100009, 1-8.

https://doi.org/10.1002/adfm.202100009. 
(34) Hu, Q.; Sun, D.; Wu, Q.; Wang, H.; Wang, L.; Liu, B.; Zhou, A.; He, J. MXene: A New Family of Promising Hydrogen Storage Medium. J. Phys. Chem. A 2013, 117 (51), 14253-14260. https://doi.org/10.1021/jp409585v.

(35) Hu, Q.; Wang, H.; Wu, Q.; Ye, X.; Zhou, A.; Sun, D.; Wang, L.; Liu, B.; He, J. TwoDimensional Sc2C: A Reversible and High-Capacity Hydrogen Storage Material Predicted by First-Principles Calculations. Int. J. Hydrogen Energy 2014, 39 (20), 1060610612. https://doi.org/https://doi.org/10.1016/j.ijhydene.2014.05.037.

(36) Rietveld, H. M. The Rietveld Method. Phys. Scr. 2014, 89 (9). https://doi.org/10.1088/0031-8949/89/9/098002.

(37) Ganose, A. M.; Park, J.; Faghaninia, A.; Woods-Robinson, R.; Persson, K. A.; Jain, A. Efficient Calculation of Carrier Scattering Rates from First Principles. arXiv 2020, No. 2021. https://doi.org/10.1038/s41467-021-22440-5.

(38) A. Louis Allred. Electronegativity Values from Thermochemical Data. J. Inorg. Nucl. Chem. 1961, 17 (1949), 215-221.

(39) Fall, C. J.; Binggeli, N.; Baldereschi, A. Deriving Accurate Work Functions from ThinSlab Calculations. J. Phys. Condens. Matter 1999, 11 (13), 2689-2696. https://doi.org/10.1088/0953-8984/11/13/006.

(40) De Jong, M.; Chen, W.; Angsten, T.; Jain, A.; Notestine, R.; Gamst, A.; Sluiter, M.; Ande, C. K.; Van Der Zwaag, S.; Plata, J. J.; et al. Charting the Complete Elastic Properties of Inorganic Crystalline Compounds. Sci. Data 2015, 2. https://doi.org/10.1038/sdata.2015.9.

(41) Druffel, D. L.; Woomer, A. H.; Kuntz, K. L.; Pawlik, J. T.; Warren, S. C. Electrons on the Surface of 2D Materials: From Layered Electrides to 2D Electrenes. $11196 \mid$ J. Mater. Chem. C 2017, 5, 11196-11213. https://doi.org/10.1039/c7tc02488f.

(42) Biesinger, M. C.; Lau, L. W. M.; Gerson, A. R.; Smart, R. S. C. Resolving Surface Chemical States in XPS Analysis of First Row Transition Metals, Oxides and Hydroxides: Sc, Ti, V, Cu and Zn. Appl. Surf. Sci. 2010, 257 (3), 887-898. https://doi.org/10.1016/j.apsusc.2010.07.086.

(43) Merrick, J. Bonding in Scandium Monosulfide a NaCl Crystal Type, Iowa State University, 1980.

(44) Barr, T. L.; Seal, S. Nature of the Use of Adventitious Carbon as a Binding Energy Standard. J. Vac. Sci. Technol. A Vacuum, Surfaces, Film. 1995, 13 (3), 1239-1246. https://doi.org/10.1116/1.579868.

(45) Ramqvist, L.; Hamrin, K.; Johansson, G.; Fahlman, A.; Nordling, C. Charge Transfer in Transition Metal Carbides and Related Compounds Studied by ESCA. J. Phys. Chem. Solids 1969, 30, 1835-1847. 